\title{
The novel p.Gly306Asp perforin mutation causes familial hemophagocytic lymphohistiocytosis type 2 (FHL-2) probably due to a critical role of Gly306 in the pore-forming perforin domain
}

\author{
Ramón Urrea Moreno ${ }^{\mathrm{a}}$, Itziar Astigarraga ${ }^{\mathrm{b}, \mathrm{c}, \mathrm{d}}$, Ana Fernández-Teijeiro ${ }^{\mathrm{e}}$, \\ Carmen Rodríguez-Sainz ${ }^{a}$, María Alonso-Martinez ${ }^{a}$, Lydia Martín-Martín ${ }^{a}$, \\ Susana García-Obregón ${ }^{c}$, and Juana Gil-Herrera ${ }^{a *}$
}

\begin{abstract}
Background: Three substitutions at either Gly305 or Gly306 within the membrane attack/complex perforin domain (MACPF) of perforin have been previously identified in a number of patients with hemophagocytic lymphohistiocytosis $(\mathrm{HLH})$. However, their pathogenic impact remains unclear since all the cases reported so far carried heterozygous genotypes and showed very heterogeneous clinical presentations. Here, we report a new substitution (p.Gly306Asp) and use in silico tools to elucidate the pathogenic mechanisms and severity associated with human Gly306 and Gly305 mutations.

Methods: The immunological workup included perforin expression and perforin gene (PRF1) mutation analysis. Computer algorithms based on conservation, secondary, and tertiary protein structure analyses were applied to assess the role of the mutations in disease pathogenesis.

Results: In our patient, we found a previously undescribed homozygous c. 917G>A (p.Gly306Asp) mutation in the PRF1 gene that was associated with null perforin expression in her natural killer lymphocytes. Sequence alignments revealed that Gly306 and Gly305 are highly conserved positions among vertebrate perforins, as well as in other related pore-forming proteins such as bacterial cytolysins. Further in silico analyses consistently predicted mutations in these 2 positions to be pathogenic due to diminished stability of the perforin molecule.

Conclusion: Age of HLH onset, severity of the disease and undetectable perforin in our p.Gly306Asp homozygous patient along with the in silico results unmask this novel mutation as highly detrimental. Our results highlight the need of combining all clinical features, in vitro phenotypes and computer based approaches to classify human perforin mutations accurately.

Statement of novelty: The study of these PRF1 mutations points to an important role of the 2 glycine amino acids (Gly305 and Gly306) in the molecular stability of perforin, which may also be likely in other pore-forming proteins. Our in silico results conclude that the pathogenicity of mutations in highly conserved Gly305 and Gly306 is likely to be associated with a serious destabilization of the native perforin conformation.
\end{abstract}

${ }^{a}$ Hospital General Universitario and Health Research Institute "Gregorio Marañón", Madrid, Spain; ' Paediatric Service, Hospital Universitario Cruces,

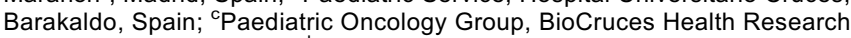
Institute, Barakaldo, Spain; ${ }^{d}$ Department of Paediatric, Faculty of Medicine and Dentistry, University of the Basque Country, UPV/EHU, Leioa, Bizkaia, Spain epediatric Onco-Hematology Unit, Hospital Virgen de la Macarena, Sevilla, Spain
Submitted 23 May 2017

Accepted 27 July 2017

Available online 3 August 2017

LymphoSign Journal 4:101-112 (2017)

dx.doi.org/10.14785/lymphosign-2017-0006 


\section{Introduction}

Familial hemophagocytic lymphohistiocytosis (FHL) is a primary immunodeficiency due to defects in several genes involved in the cytotoxic pathway (Janka 2012; Sieni et al. 2014). FHL usually presents during early childhood and is fatal without hematopoietic cell transplantation (HCT); however, atypical, late-onset FHL cases are increasingly being described (Clementi et al. 2002; Molleran Lee et al. 2004; Mancebo et al. 2006; Zur Stadt et al. 2006; Nagafuji et al. 2007; Ueda et al. 2007; Zhang et al. 2011; Sanchez et al. 2012; Sieni et al. 2012; Wang et al. 2012; Dias et al. 2013; Mhatre et al. 2014; Tesi et al. 2015; Barmettler et al. 2016; Gao et al. 2016).

Type 2 FHL (FHL-2) is caused by mutations in the PRF1 gene (Stepp et al. 1999; Voskoboinik et al. 2010; Voskoboinik et al. 2015). PRF1 encodes perforin, a protein present in the secretory granules of cytotoxic $\mathrm{T}$ and natural killer (NK) lymphocytes, which is essential for cytotoxic immune responses and homeostasis (Voskoboinik et al. 2010; Thiery and Lieberman 2014; Voskoboinik et al. 2015). Most of the mutations reported in FHL-2 patients are located in the membrane attack complex/perforin (MACPF) domain (An et al. 2013; Voskoboinik et al. 2015). The MACPF domain is critical for pore formation, specifically the oligomerization of perforin molecules after their preactivation by binding to the target cell membrane (Baran et al. 2009; Stewart et al. 2015; Yagi et al. 2015). Despite recent advances regarding perforin structure (Law et al. 2010; Yagi et al. 2015), little is known about the precise mechanism of pore formation by perforin, and the molecular mechanisms underlying the pathogenicity of MACPF mutations remain poorly understood.

We describe an early-onset FHL-2 case showing the novel MACPF homozygous missense mutation c. $917 \mathrm{G}>\mathrm{A}$ which leads to the amino acid change p.Gly306Asp. Different amino acid substitutions that are located at the same position (c.916G $>\mathrm{T}$ leading to p.Gly306Cys (Grossman et al. 2005; Abdalgani et al. 2015), c.916G > A leading to p.Gly306Ser (Nagafuji et al. 2007; Wang et al. 2012; Gao et al. 2016)) or neighbouring glycine (c.914C $>\mathrm{A}$, leading to p.Gly305Asp, (Clementi et al. 2005)) have been previously reported in very heterogeneous FHL-2 patients. We performed perforin immunophenotyping and in silico analysis to ascertain the relevance of the novel
p.Gly306Asp as well as the above mentioned mutations in HLH pathogenesis.

\section{Functional and clinical presentation}

Case report: A 19-month-old girl, the first child of a consanguineous couple with no previous familial history of hemophagocytic lympohistiocytosis (HLH), was first admitted to hospital because of fever due to unknown origin. Progressive anemia (haemoglobin $7.9 \mathrm{~g} / \mathrm{L})$, thrombopenia $(8000 / \mu \mathrm{l})$, hypertrigliceridemia $(720 \mathrm{mg} / \mathrm{dL})$, elevated ferritin $(3373 \mathrm{ng} / \mathrm{mL})$, hypofibrinoginemia $(120 \mathrm{mg} / \mathrm{dL})$ and hepatosplenomegaly, along with hemophagocytosis in bone marrow led to diagnosis of HLH, and she was started on the HLH-2004 therapeutic protocol (Henter et al. 2007). According to the immunological results, she was classified as FHL-2 and after HLH-2004 induction therapy and conditioning, she underwent cord blood HCT $\left(1.36 \times 10^{5} \mathrm{CD} 34^{+}\right.$ cells). Continuing maintenance treatment was required post-HCT and the patient unfortunately died at 150 days post-transplant because of graft failure and HLH disease reactivation.

Perforin expression was assessed by flow cytometry using anti-T cell receptor FITC, anti-CD8 PerCP, and anti-CD56 APC (BD Biosciences, San Jose, CA) antibodies for surface staining and anti-perforin $\mathrm{PE}$ (clone DG9, Pharmingen, BD Bioscience) or anti-granzyme B PE (Immunotech) antibodies for intracellular staining, as previously described (Urrea Moreno et al. 2009). Samples were acquired in a FACSCalibur cytometer and analysed with FlowJo 7.6.1 software. Figure 1A shows that perforin staining within patient's circulating NK lymphocytes (corresponding to $16 \%$ of total lymphocytes) was as negative as the fluorescence intensity of isotypic control. On the other hand, granzyme-B expression-analyzed as a positive intracellular staining-was increased in the patient when compared to the patient's mother and a healthy individual tested the same day of the analysis (Figure 1B).

The mutational analysis was performed on genomic DNA from peripheral blood. Coding region of PRF1 (exons 2 and 3) was amplified by PCR (Gene Amp PCR System 9700, PE Applied Biosystems, Warrington, UK) and sequenced (ABI PRISM 310 Genetic Analyzer from Applied Biosystems) as described (Urrea Moreno et al. 2009). Chromatograms were created with Geneious Pro 5.5.7 and sequences were compared with 

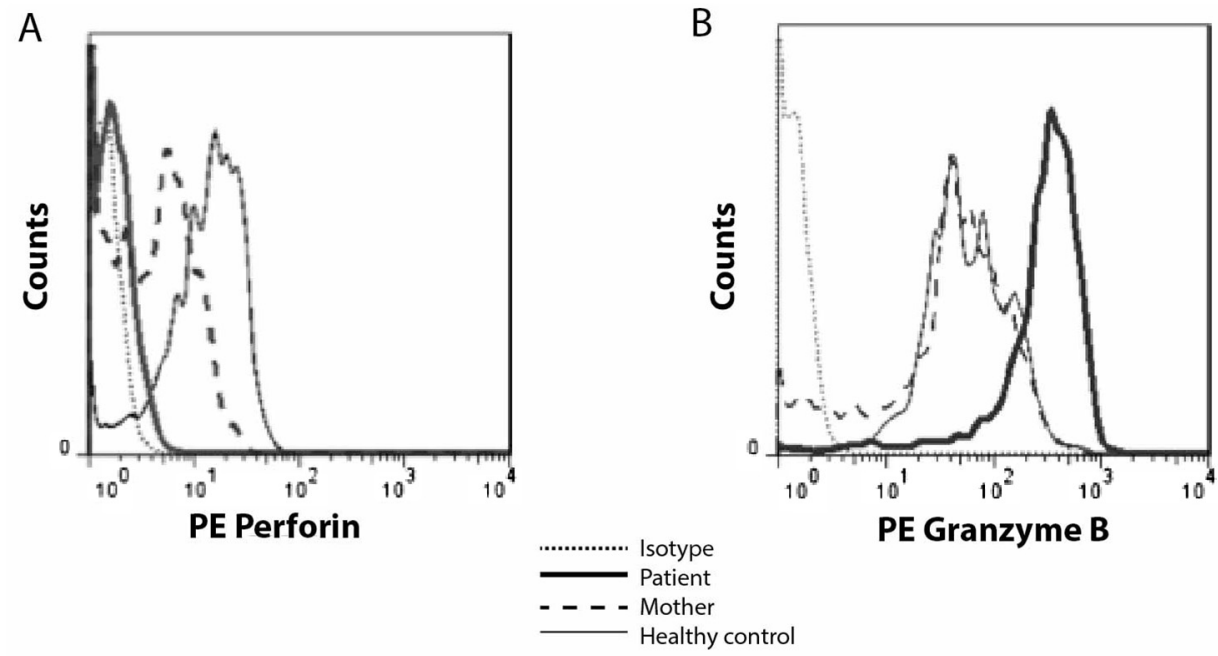

C

Normal control (WT)

Patient (c.917G>A)
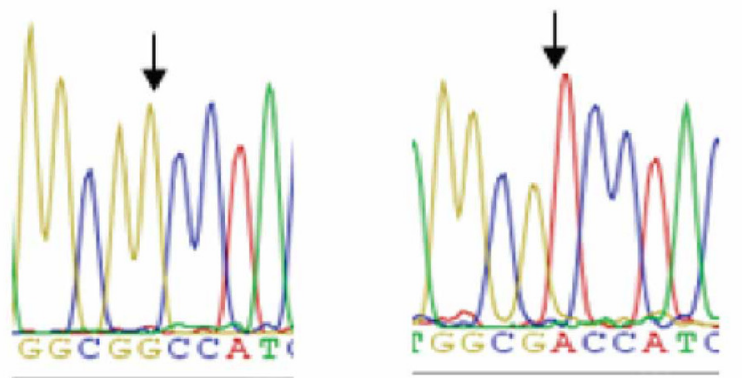

Father (WT/c.917G >A)

Mother (WT/c.917G > A)
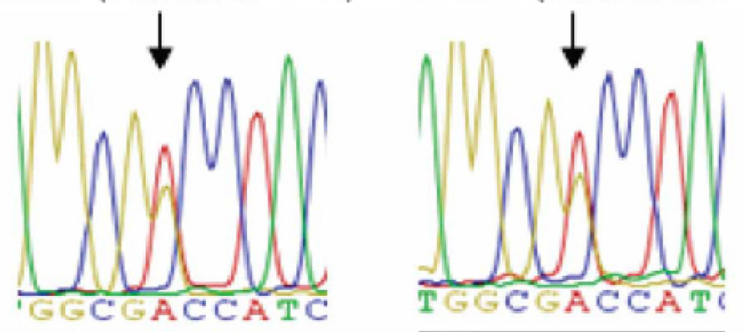

Figure 1: Intracellular protein expression and PRF1 sequencing. Flow cytometry was used to detect perforin $(A)$ and granzyme-B $(B)$ in NK-cells from patient, her mother, and a healthy control. Both histograms are gated on $\mathrm{CD}^{+} 6^{+} \mathrm{TCRab}^{-}$cells. The counts of positive gated events represented on the $Y$ axis included at least 500 to a maximum of 1600 cells. (C) DNA electropherograms. Arrows indicate the position at the wild-type PRF1 gene sequence in exon 3 , and the homozygous missense mutation at the corresponding position in the patient as well as the heterozygous change in her parents.

reference PRF1 (RefSeq: NG_009615.1). Figure 1C shows the homozygous mutation c. $917 \mathrm{G}>\mathrm{A}$ in exon 3 of PRF1 that was found in the patient; this nucleotide change results in the non-conservative substitution of the non-polar amino acid glycine at position 306 of wild-type perforin protein with the negatively charged aspartic acid (p.Gly306Asp) in MACPF domain. The parents were both found to be heterozygous carriers of the mutated allele (Figure 1C), correlating with the decreased perforin expression found in the mother (Figure 1A).

Figure 2 shows a molecular model of perforin and of perfringolysin (PFO), a bacterial cholesterol dependant cytolysin (CDC), to illustrate the structural homology of their 2 pore-forming domains. 3D models were performed with Yasara View, v16.7.22 (http://yasara.org/) (Krieger and Vriend 2014). Gly306 and the preceding 

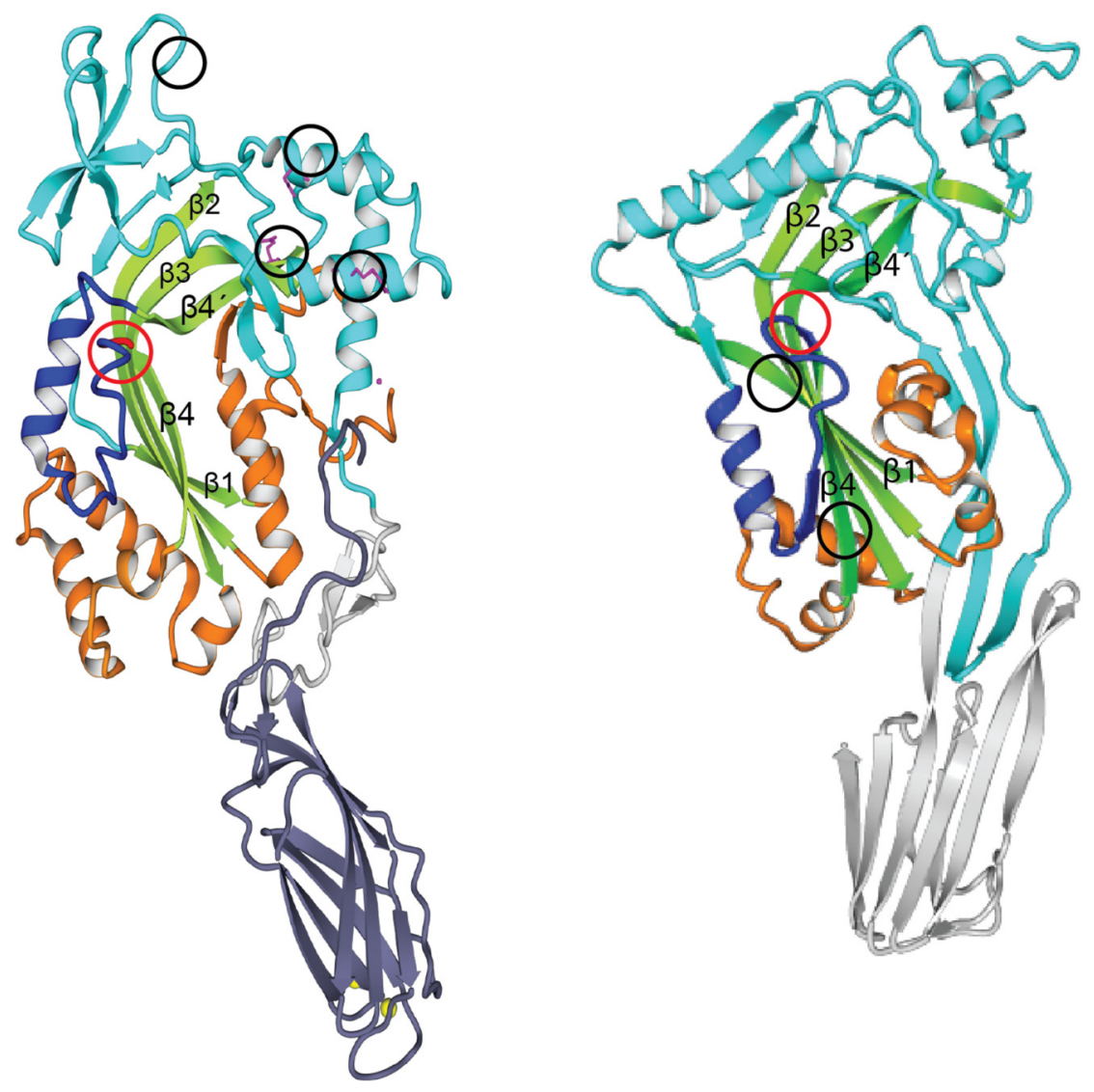

Figure 2: Molecular models of perforin and PFO. Ribbon representations of perforin molecule on the left, based on the murine perforin crystal structure (PDB identifier: 3nsj) and the cytolysin PFO (PDB identifier:1pfo) on the right, are shown. Different subregions of pore forming domains were coloured as follows: in green, the antiparallel central $\beta$ sheet (conformed by $\beta 1, \beta 2, \beta 3, \beta 4$, and $\beta 4$ '); in dark blue, the "U" shaped helical loop connecting $\beta 4$ and $\beta 4^{\prime}$ regions, with Gly305 and Gly306 in perforin, or the homologous glycines in PFO circled in red; in orange, clusters of transmembrane helices $\mathrm{CH} 1$ and $\mathrm{CH} 2$; in cyan, remaining MACPF regions. Circled in black on each protein are the location of residues potentially involved in perforin or PFO oligomerization.

Gly305 are located at the beginning of a helical loop region that connects $\beta 4$ and $\beta 4^{\prime}$ strands of the central beta sheet in both MACPFs and CDCs (Lukoyanova et al. 2016; Dudkina et al. 2016). As shown in Figure 2, no proximity exists between the helical loop in perforin and the putative residues involved in its oligomerization, as opposed to what has been previously described in PFO (Ramachandran et al. 2004).

For amino acid conservation analysis, representative sequences were obtained from the NCBI database (www.ncbi.nlm.nih.gov). Alignments were performed with PROMALS3D (http://prodata.swmed.edu/ promals3d/promals3d.php), and edited with Geneious Pro 5.5.7 using Blosum45 matrix. Figure 3 shows that both Gly305 and Gly306 in human perforin molecule or corresponding glycines in other vertebrate perforins, as well as other MACPF domains from different proteins, or even in pore-forming domains of bacterial cholesterol CDCs, constitute a highly conserved motif in all these proteins despite the low global sequence homology.

This strikingly high conservation led us to hypothesize that substitutions in any of these 2 glycines would be functionally detrimental for perforin, and therefore pathogenic. To predict the pathogenicity of mutations, we used Polyphen-2 server, version 2.2.2 (http:// genetics.bwh.harvard.edu/pph2/), that collects information available on conservation, secondary and tertiary structure of the molecule, as well as functional sites 


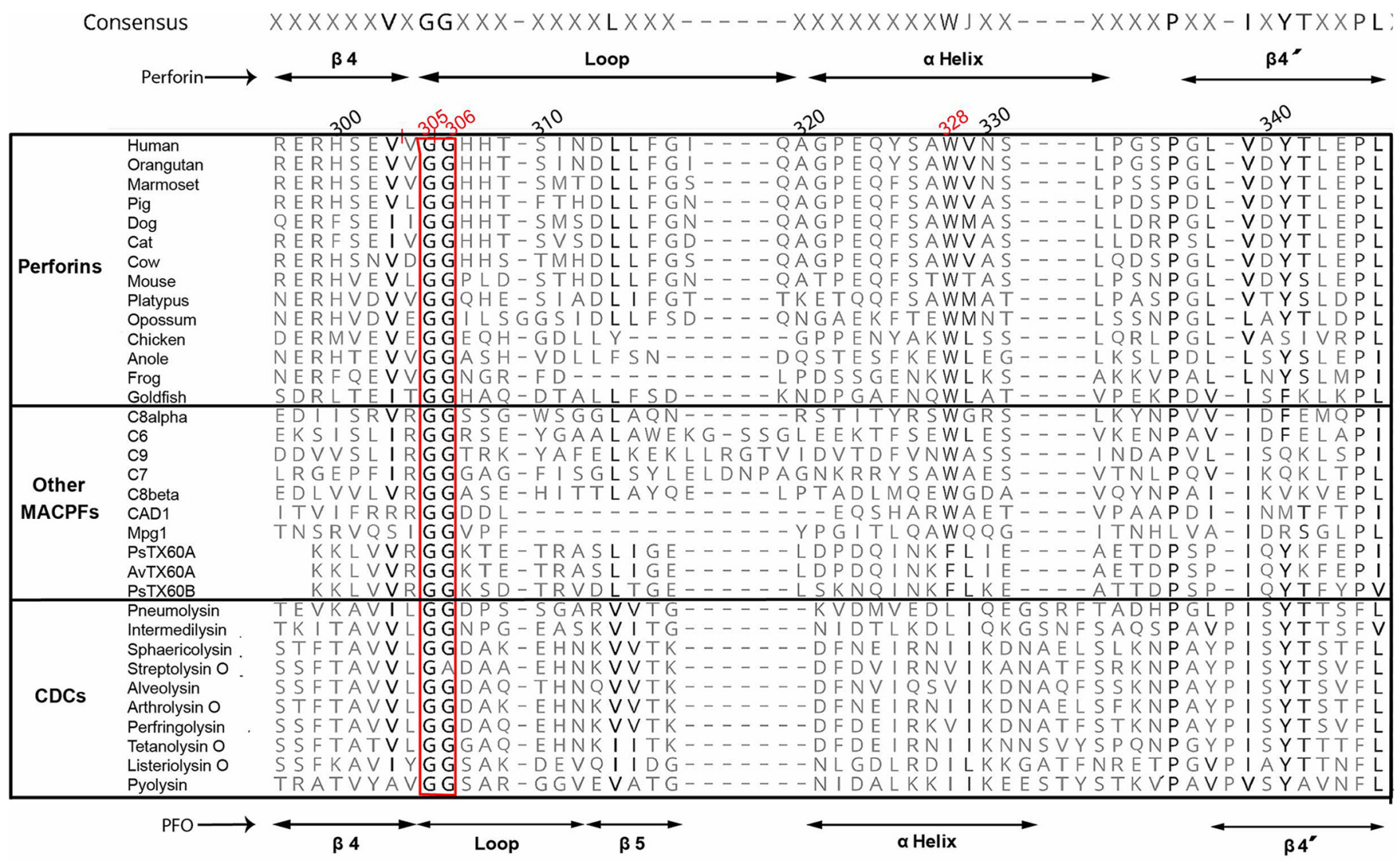

Figure 3: Conservation of the glycine pair across vertebrate perforins, other protein containing MACPFs and CDCs. Shown are sequences corresponding to the region including $\beta 4$, the helical loop and $\beta 4^{\prime}$. The glycine motif is enclosed in the red column. Consensus sequence is shown at the top of the alignment and it is based on the rule of $50 \%$ identity. Amino acid numbering corresponds to human perforin, with Gly305, Gly306, and Trp328 indicated in red. Secondary structures corresponding to MACPFs (as represented by perforin) are indicated at the top of the alignment; secondary structures corresponding to CDCs (as represented by PFO) are indicated at the bottom of the alignment. Name of the species (vertebrate perforins) or proteins (other MACPFs, CDCs) is shown preceding the corresponding sequence. Higher degree of amino acid conservation is denoted by higher grey intensity.

hypothetically altered by a new residue (Adzhubei et al. 2010). As shown in Table 1, p.Gly306Asp, as well as p.Gly305Asp (Clementi et al. 2005), Gly306Cys (Grossman et al. 2005; Abdalgani et al. 2015) and p.Gly306Ser (Nagafuji et al. 2007; Wang et al. 2012; Gao et al. 2016) were undoubtedly predicted as pathogenic by the HuVar strategy of Polyphen-2.

We next studied the impact of the above mutations on perforin thermodynamic stability, or the change in folding free energy upon mutation (i.e., differences in Gibb's free energy between the folded and unfolded state of mutant compared to wild-type molecules, also $\Delta \Delta \mathrm{G}$ or ddG). For this, we generated a molecular model of human perforin, using BuildModel and RepairPDB commands within FoldX v4 (http://foldxsuite.crg.eu) (Guerois et al. 2002), employing perforin X-ray crystal structure (PDB identifier:3nsj) (Law et al. 2010) as a template. Independent algorithms used for stability calculations were FoldX itself, SDM (http://mordred.bioc. cam.ac.uk/ sdm/sdm.php) (Worth et al. 2011), PoPMuSiC (http://dezyme.com/) (Dehouck et al. 2009) and Eris (http://troll.med.unc.edu/eris) (Yin et al. 2007). In support of our hypothesis, all 4 algorithms predicted that p.Gly306Asp, as well as the other 3 FHL-2 mutations reported in these glycines, i.e., p.Gly305Asp, p.Gly306Cys and p.Gly306Ser result in severe destabilization of the perforin molecule (Table 1), albeit to a lesser extent in p.Gly306Ser according to Eris prediction. As shown in Figure 4, Asp306 creates important steric clashes with Gly305 and Trp328, and perturbs the hydrogen bond (H-bond) between wild-type (WT)-Gly305 and WT-Trp328. The latter residue extends from the neighboring helix and 
Table 1: In silico analysis of FHL-2 associated mutations in Gly306 and Gly305.

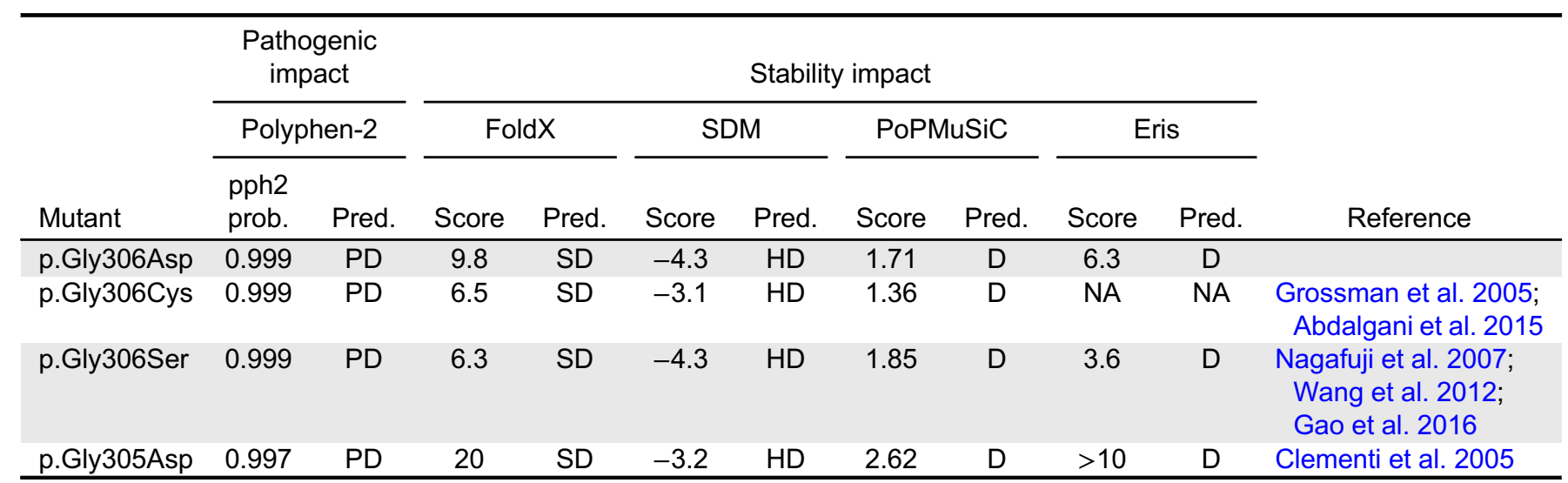

Note: Shown is the impact predicted by Polyphen-2 and several stability based in silico methods. pph2 prob.: posterior probability for being damaging; it ranges from 0 (benign) to 0.999 (most probably damaging, PD). Score: it represents the change in folding free energy upon mutation ("ddG", Kcal/mol); Pred: predicted outcome of mutations, defined as "destabilizing" (D) scores from 0 to 5 , versus "severely destabilizing" (SD) scores $>5$, in FoldX; "destabilizing" (D) scores from -0.5 to -2 , versus "highly destabilizing" (HD) scores <-2 in SDM; or simply "destabilizing" (D) scores $>0$ in PoPMuSiC and Eris. NA: not available. Options were set as follows: for FoldX, $\mathrm{pH}=7.4$, temperature $=310 \mathrm{~K}$, ion strength $=0.05 \mathrm{M}$, vdwDesign $=2$; For SDM and PoPMuSiC, default settings; for Eris, fixed backbone and no backbone pre-relaxation.
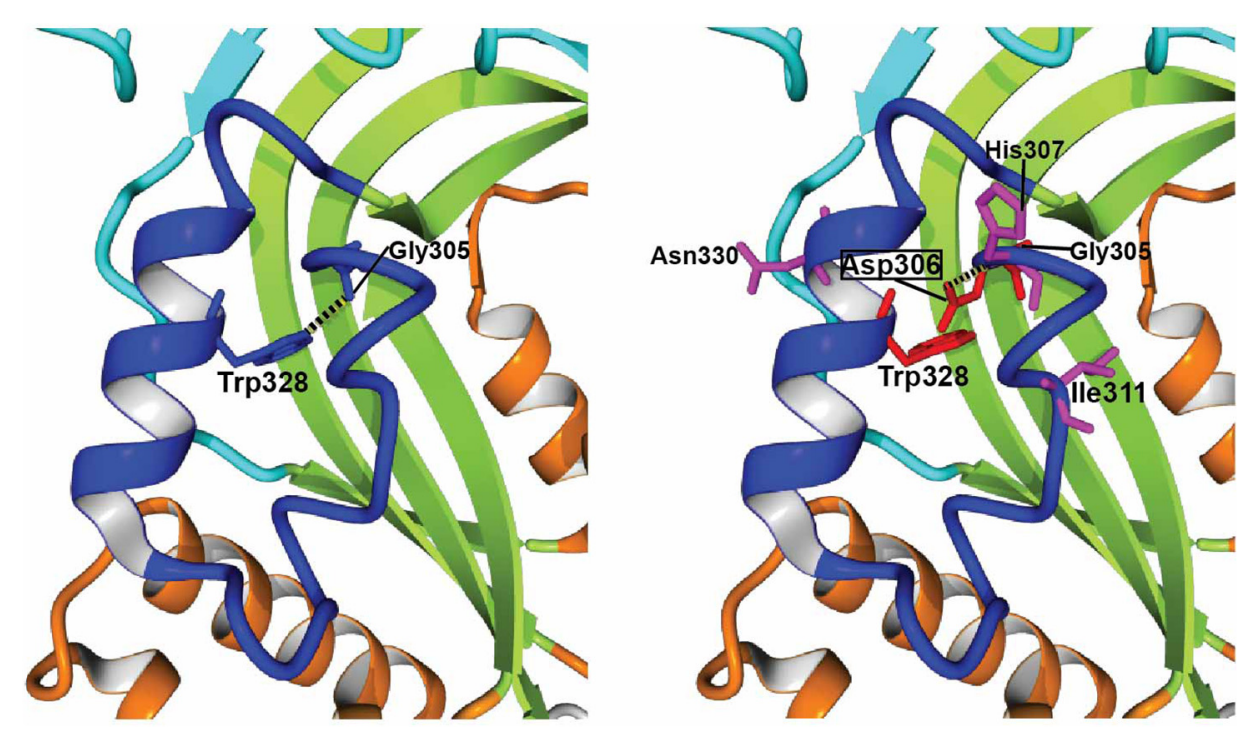

Figure 4: FoldX analysis of p.Gly306Asp perforin mutation. Shown is a zoomed view of the helical loop region of our WT (left) and p.Gly306Asp (right) human perforin models. $\mathrm{H}$-bonds are represented as black dots; in the p.Gly306Asp model (right) the H-bond from WT perforin disappears and a new $\mathrm{H}$-bond is created by the mutant. Those residues involved in steric clashes are indicated in red. Other residues affected by the mutation are coloured in magenta. Graphics performed with Yasara View v16.7.22.

is highly conserved both in vertebrate perforins and in other MACPF proteins (Figure 3), which suggests that the H-bond with WT-Gly305 or equivalent glycine plays a critical role in stability. Moreover, p.Gly306Asp originates a new H-bond between Asp306 and His307, which is absent in the WT structure. With these and other mutation-specific alterations not mentioned above, it is very likely that these 3 substitutions, and probably nearly all Gly306 variants, would be deleterious for perforin folding and (or) function.

\section{Discussion}

Intracellular perforin staining was studied as part of the current laboratory diagnostic workup in HLH patients (Arico et al. 2002; Henter et al. 2007; 
Bryceson et al. 2012; Imashuku 2014). We found null perforin expression and overexpressed granzyme-B; this granzyme pattern most likely indicates hyperactivation, given it has been previously described in the HLH setting (Molleran Lee et al. 2004; Mellor-Heineke et al. 2013). In our analysis, both the healthy donor and the patient's mother (healthy heterozygous p.Gly306Asp carrier) had no inflammatory conditions and showed normal granzyme-B expression.

Null perforin expression in our homozygous patient and the decreased, intermediate amount of perforin in her heterozygous mother along with the c. $917 \mathrm{G}>\mathrm{A}$ segregation in this family suggested the inability of p.Gly306Asp perforin to fold properly. Impaired folding would cause reduced intracellular levels and (or) conformational changes that could inhibit DG9 antibody binding. Two other mutations affecting the same amino acid position (p.Gly306Cys (Grossman et al. 2005; Abdalgani et al. 2015) and p.Gly306Ser (Nagafuji et al. 2007; Wang et al. 2012; Gao et al. 2016)) are also associated with null or very limited perforin expression in cytotoxic lymphocytes. Although diminished expression suggests misfolding for all of these mutants, a defect in perforin content could also be attributed to the altered immunoregulatory state of the active HLH setting (Voskoboinik et al. 2010). Unfortunately, we have not tested intracellular perforin in highly effective recombinant expression systems and (or) under temperatures permissive for folding as it has been done in the neighbouring mutation p.Gly305Asp (Risma et al. 2006; Chia et al. 2009; Urrea Moreno et al. 2009).

Our results show an extremely high conservation of the Gly305/Gly306 pair through MACPF/CDC superfamily, which denotes a critical role for these residues. Previous studies attributed these 2 glycines the role of a "hinge point" which allow the conformational changes leading to membrane spanning (Rosado et al. 2007; Gilbert et al. 2013; Dudkina et al. 2016; Lukoyanova et al. 2016). Although this hinge point has also been involved in the oligomerization process of the cytolysin PFO (Ramachandran et al. 2004; Rosado et al. 2007), this seems unlikely in perforin, where residues critically involved in oligomerization (Baran et al. 2009; Dudkina et al. 2016) are neither proximal nor directly connected to these glycines (Figure 2). However, since the hinge points of each subunit come into close proximity during pore formation, mutations in Gly305 and Gly306 could hypothetically perturb the association of monomers within the pore and therefore alter its properties and (or) hinder its formation.

In addition to the high conservation of both Gly305 and Gly306, Polyphen-2 predicted a severe pathogenic impact of the novel p.Gly306Asp, and of p.Gly306Cys, p.Gly306Ser and adjacent p.Gly305Asp. The 4 mutations result in high thermodynamic destabilization, as predicted by the 4 algorithms that we have used. The impact on stability would primarily explain HLH pathogenicity associated to these mutations. Decreased stability is a major factor underlying the dysfunction of disease-associated missense mutations (Wang and Moult 2001; Yue et al. 2005; Pey et al. 2007; Casadio et al. 2011; Stefl et al. 2013). A recent and comprehensive in silico study of human perforin molecule, using FoldX and PoPMUSIC as predictive algorithms, identified nearly half of all missense FHL-2 perforin mutations as significantly destabilizing (An et al. 2013). Gly305Asp and Gly306Cys were also included in this work, and their predicted impact was nearly identical to what we have found with our human perforin model.

Protein destabilization results in misfolding and premature degradation, and correlates positively with the degree of protein dysfunction and clinical severity (Pey et al. 2007; Tokuriki et al. 2008; Tokuriki and Tawfik 2009; McKeone et al. 2014). Interestingly, the Arginine mutation of Gly357 in the complement factor C7-a glycine corresponding to Gly305 in perforin, is one of the most frequent mutations associated with C7 deficiency, and was linked to serious misfolding and defective secretion (Fernie et al. 1997; Barroso et al. 2004; Rameix-Welti et al. 2007; Barroso et al. 2010).

Perforin immunophenotyping and the predictions in silico suggest serious misfolding in the novel p.Gly306Asp, as well as the p.Gly306Cys, p.Gly306Ser and the p.Gly305Asp mutants. As discussed earlier, it may be possible that residual perforin expression and secretion of those mutants could still exist when using recombinant mutants in in vitro expression systems. However, even if secreted, it is likely that the mutants would display aberrant pore formation, since the Gly305/306 pair is presumably essential for this postsynaptic process. 
Our in silico methods homogeneously predicted a severe clinical impact of Gly305/Gly306 FHL-2 mutations. This correlates with the early onset FHL-2 in our patient with homozygous p.Gly306Asp mutation, along with the very early onset and undetectable NKcell mediated killing FHL-2 in 2 triplets with p.Gly306Cys mutation (Grossman et al. 2005). However, less severity could be attributed to p.Gly306Ser, found in 2 late onset FHL-2 patients and even in a healthy sibling; all of them carrying compound heterozygous $P R F 1$ genotypes (Nagafuji et al. 2007; Gao et al. 2016). In fact, despite its impaired maturation, likely related to misfolding, p.Gly306Ser mutant seems to retain cytotoxic function (Nagafuji et al. 2007). Finally, p.Gly305Asp was described along with the p.Arg356Trp in an 18 year-old HLH patient (Clementi et al. 2005). Since p.Arg356Trp is considered a hypomorphic mutant (Zhang et al. 2011; Huang et al. 2014), this may explain the delayed age of HLH onset in this patient.

In summary, HLH in our patient was caused by a unique, homozygous mutation p.Gly306Asp in a critical residue located at MACPF domain of perforin. Age of onset and nearly absent perforin expression pointed to p.Gly306Asp being a severe mutant, which is further supported by our in silico studies. In vitro studies with recombinant expressed mutants would be needed to definitively classify p.Gly306Ser and related FHL-2 mutations, and to determine the precise contribution of the proposed misfolding (pre-synaptic) and (or) the inhibition of pore formation (post-synaptic) as the molecular factors underlying their pathogenicity (Voskoboinik et al. 2010).

\section{Funding}

This work was supported by a grant from the Spanish NIH (ISCIII FIS PI12/02761).

\section{Conflict of interest}

The authors declare no conflict of interest.

\section{Acknowledgements}

The authors thank Professor Andrew Herr for reviewing the manuscript and for his useful advice on discussing the in silico results. We are also grateful to Jane Kinnear for technical help.

\section{REFERENCES}

Abdalgani, M., Filipovich, A.H., Choo, S., Zhang, K., Gifford, C., Villanueva, J., Bleesing, J.J., and Marsh, R.A. 2015. Accuracy of flow cytometric perforin screening for detecting patients with FHL due to PRF1 mutations. Blood. 126(15):1858-1860. PMID: 26450956. doi: 10.1182/blood-2015-06648659.

Adzhubei, I.A., Schmidt, S., Peshkin, L., Ramensky, V.E., Gerasimova, A., Bork, P., Kondrashov, A.S., and Sunyaev, S.R. 2010. A method and server for predicting damaging missense mutations. Nat. Methods. 7(4):248-249. PMID: 20354512. doi: 10.1038/ nmeth0410-248.

An, O., Gursoy, A., Gurgey, A., and Keskin, O. 2013. Structural and functional analysis of perforin mutations in association with clinical data of familial hemophagocytic lymphohistiocytosis type 2 (FHL2) patients. Protein Sci. 22(6):823-839. PMID: 235 92409. doi: 10.1002/pro.2265.

Arico, M., Allen, M., Brusa, S., Clementi, R., Pende, D., Maccario, R., Moretta, L., and Danesino, C. 2002. Haemophagocytic lymphohistiocytosis: Proposal of a diagnostic algorithm based on perforin expression. Br. J. Haematol. 119(1):180-188. PMID: 12358924. doi: 10.1046/j.1365-2141.2002. 03773.x.

Baran, K., Dunstone, M., Chia, J., Ciccone, A., Browne, K.A., Clarke, C.J., Lukoyanova, N., Saibil, H., Whisstock, J.C., Voskoboinik, I., and Trapani, J.A. 2009. The molecular basis for perforin oligomerization and transmembrane pore assembly. Immunity. 30(5):684-695. PMID: 194 46473. doi: 10.1016/j.immuni.2009.03.016.

Barmettler, S., Nowak, R.J., Parker, T., and Price, C. 2016. Previously undiagnosed fatal familial haemophagocytic lymphohistiocytosis in a 24 -year-old woman. BMJ Case Rep. 2016. doi: 10.1136/bcr2015-213698.

Barroso, S., Lopez-Trascasa, M., Merino, D., Alvarez, A.J., Nunez-Roldan, A., and Sanchez, B. 2010. C7 deficiency and meningococcal infection susceptibility in two spanish families. Scand. J. Immunol. 72(1):38-43. PMID: 20591074. doi: 10. 1111/j.1365-3083.2010.02403.x.

Barroso, S., Sanchez, B., Alvarez, A.J., Lopez-Trascasa, M., Lanuza, A., Luque, R., Wichmann, I., and NunezRoldan, A. 2004. Complement component C7 deficiency in two Spanish families. Immunology. 113(4):518-523. PMID: 15554930. doi: 10.1111/j. 1365-2567.2004.01997.x. 
Bryceson, Y.T., Pende, D., Maul-Pavicic, A., Gilmour, K.C., Ufheil, H., Vraetz, T., Chiang, S.C., Marcenaro, S., Meazza, R., Bondzio, I., Walshe, D., Janka, G., Lehmberg, K., Beutel, K., zur Stadt, U., Binder, N., Arico, M., Moretta, L., Henter, J.I., and Ehl, S. 2012. A prospective evaluation of degranulation assays in the rapid diagnosis of familial hemophagocytic syndromes. Blood. 119(12):2754-2763. PMID: 22294731. doi: 10.1182/blood-2011-08-374199.

Casadio, R., Vassura, M., Tiwari, S., Fariselli, P., and Luigi Martelli, P. 2011. Correlating disease-related mutations to their effect on protein stability: A largescale analysis of the human proteome. Hum. Mutat. 32(10):1161-1170. PMID: 21853506. doi: 10.1002/ humu.21555.

Chia, J., Yeo, K.P., Whisstock, J.C., Dunstone, M.A., Trapani, J.A., and Voskoboinik, I. 2009. Temperature sensitivity of human perforin mutants unmasks subtotal loss of cytotoxicity, delayed FHL, and a predisposition to cancer. Proc. Natl. Acad. Sci. USA. 106(24):9809-9814. PMID: 19487666. doi: 10.1073/ pnas.0903815106.

Clementi, R., Emmi, L., Maccario, R., Liotta, F., Moretta, L., Danesino, C., and Arico, M. 2002. Adult onset and atypical presentation of hemophagocytic lymphohistiocytosis in siblings carrying PRF1 mutations. Blood. 100(6):2266. PMID: 12229880. doi: 10.1182/blood-2002-04-1030.

Clementi, R., Locatelli, F., Dupre, L., Garaventa, A., Emmi, L., Bregni, M., Cefalo, G., Moretta, A., Danesino, C., Comis, M., Pession, A., Ramenghi, U., Maccario, R., Arico, M., and Roncarolo, M.G. 2005. A proportion of patients with lymphoma may harbor mutations of the perforin gene. Blood. 105(11):44244428. PMID: 15728124. doi: 10.1182/blood-200404-1477.

Dehouck, Y., Grosfils, A., Folch, B., Gilis, D., Bogaerts, P., and Rooman, M. 2009. Fast and accurate predictions of protein stability changes upon mutations using statistical potentials and neural networks: PoPMuSiC-2.0. Bioinformatics. 25(19):2537-2543. PMID: 19654118. doi: 10.1093/bioinformatics/btp445.

Dias, C., McDonald, A., Sincan, M., Rupps, R., Markello, T., Salvarinova, R., Santos, R.F., Menghrajani, K., Ahaghotu, C., Sutherland, D.P., Fortuno, E.S., III, Kollmann, T.R., Demos, M., Friedman, J.M., Speert, D.P., Gahl, W.A., and Boerkoel, C.F. 2013. Recurrent subacute post-viral onset of ataxia associated with a PRF1 mutation. Eur. J. Hum. Genet. 21:1232-1239. doi: 10.1038/ ejhg.2013.20.
Dudkina, N.V., Spicer, B.A., Reboul, C.F., Conroy, P.J., Lukoyanova, N., Elmlund, H., Law, R.H., Ekkel, S.M., Kondos, S.C., Goode, R.J., Ramm, G., Whisstock, J.C., Saibil, H.R., and Dunstone, M.A. 2016. Structure of the poly-C9 component of the complement membrane attack complex. Nat. Commun. 7:10588. doi: 10.1038/ ncomms 10588 .

Fernie, B.A., Orren, A., Sheehan, G., Schlesinger, M., and Hobart, M.J. 1997. Molecular bases of C7 deficiency: Three different defects. J. Immunol. 159(2):1019-1026. PMID: 9218625.

Gao, L., Dang, X., Huang, L., Zhu, L., Fang, M., Zhang, J., Xu, X., Li, T., Zhao, L., Wei, J., and Zhou, J. 2016. Search for the potential "second-hit" mechanism underlying the onset of familial hemophagocytic lymphohistiocytosis type 2 by whole-exome sequencing analysis. Transl. Res. 170:26-39. PMID: 26739415. doi: 10.1016/j.trsl.2015.12.004.

Gilbert, R.J., Mikelj, M., Dalla Serra, M., Froelich, C.J., and Anderluh, G. 2013. Effects of MACPF/CDC proteins on lipid membranes. Cell Mol. Life Sci. 70(12):2083-2098. PMID: 22983385. doi: 10.1007/ s00018-012-1153-8.

Grossman, W.J., Radhi, M., Schauer, D., Gerday, E., Grose, C., and Goldman, F.D. 2005. Development of hemophagocytic lymphohistiocytosis in triplets infected with HHV-8. Blood. 106(4):1203-1206. PMID: 15840696. doi: 10.1182/blood-2005-030950.

Guerois, R., Nielsen, J.E., and Serrano, L. 2002. Predicting changes in the stability of proteins and protein complexes: A study of more than 1000 mutations. J. Mol. Biol. 320(2):369-387. PMID: 12079393. doi: 10.1016/S0022-2836(02)00442-4.

Henter, J.I., Horne, A., Arico, M., Egeler, R.M., Filipovich, A.H., Imashuku, S., Ladisch, S., McClain, K., Webb, D., Winiarski, J., and Janka, G. 2007. HLH-2004: Diagnostic and therapeutic guidelines for hemophagocytic lymphohistiocytosis. Pediatr. Blood Cancer. 48(2):124-131. PMID: 16937360. doi: 10.1002/pbc.21039.

Huang, Z., Wang, Y., Xie, Z., and Shen, K. 2014. Remission and relapse of hemophagocytic lymphohistiocytosis in a patient harboring a PRF1 homozygous mutation: A case report. J. Pediatr. Hematol. Oncol. 36(1):e5-e8. PMID: 23073044. doi: 10.1097/ MPH.0b013e318271c963.

Imashuku, S. 2014. Hemophagocytic lymphohistiocytosis: Recent progress in the pathogenesis, diagnosis and treatment. World J. Hematol. 3(3):14. doi: 10.5315/ WJH.v3.i3.71. 
Janka, G.E. 2012. Familial and acquired hemophagocytic lymphohistiocytosis. Annu. Rev. Med. 63:233246. PMID: 22248322. doi: 10.1146/annurev-med041610-134208.

Krieger, E., and Vriend, G. 2014. YASARA ViewMolecular graphics for all devices-From smartphones to workstations. Bioinformatics. 30(20):2981-2982. PMID: 24996895. doi: 10.1093/ bioinformatics/btu426.

Law, R.H., Lukoyanova, N., Voskoboinik, I., CaradocDavies, T.T., Baran, K., Dunstone, M.A., D’Angelo, M.E., Orlova, E.V., Coulibaly, F., Verschoor, S., Browne, K.A., Ciccone, A., Kuiper, M.J., Bird, P.I., Trapani, J.A., Saibil, H.R., and Whisstock, J.C. 2010. The structural basis for membrane binding and pore formation by lymphocyte perforin. Nature. 468(7322):447-451. PMID: 21037563. doi: 10.1038/ nature09518.

Lukoyanova, N., Hoogenboom, B.W., and Saibil, H.R. 2016. The membrane attack complex, perforin and cholesterol-dependent cytolysin superfamily of poreforming proteins. J. Cell Sci. 129(11):2125-2133. PMID: 27179071. doi: 10.1242/jcs.182741.

Mancebo, E., Allende, L.M., Guzman, M., Paz-Artal, E., Gil, J., Urrea-Moreno, R., Fernandez-Cruz, E., Gaya, A., Calvo, J., Arbos, A., Duran, M.A., Canet, R., Balanzat, J., Udina, M.A., and Vercher, F.J. 2006. Familial hemophagocytic lymphohistiocytosis in an adult patient homozygous for $\mathrm{A} 91 \mathrm{~V}$ in the perforin gene, with tuberculosis infection. Haematologica. 91(9):1257-1260. PMID: 16956828.

McKeone, R., Wikstrom, M., Kiel, C., and Rakoczy, P.E. 2014. Assessing the correlation between mutant rhodopsin stability and the severity of retinitis pigmentosa. Mol. Vis. 20:183-199. PMID: 24520188.

Mellor-Heineke, S., Villanueva, J., Jordan, M.B., Marsh, R., Zhang, K., Bleesing, J.J., Filipovich, A.H., and Risma, K.A. 2013. Elevated granzyme B in cytotoxic lymphocytes is a signature of immune activation in hemophagocytic lymphohistiocytosis. Front. Immunol. 472. doi: 10.3389/fimmu.2013.00072.

Mhatre, S., Madkaikar, M., Jijina, F., and Ghosh, K. 2014. Unusual clinical presentations of familial hemophagocytic lymphohistiocytosis type-2. J. Pediatr. Hematol. Oncol. 36(8):e524-e527. PMID: 24390453. doi: 10.1097/MPH.0000000000000102.

Molleran Lee, S., Villanueva, J., Sumegi, J., Zhang, K., Kogawa, K., Davis, J., and Filipovich, A.H. 2004. Characterisation of diverse PRF1 mutations leading to decreased natural killer cell activity in North American families with haemophagocytic lymphohistiocytosis. J. Med. Genet. 41(2):137-144. PMID: 14757862. doi: 10.1136/jmg.2003.011528.

Nagafuji, K., Nonami, A., Kumano, T., Kikushige, Y., Yoshimoto, G., Takenaka, K., Shimoda, K., Ohga, S., Yasukawa, M., Horiuchi, H., Ishii, E., and Harada, M. 2007. Perforin gene mutations in adult-onset hemophagocytic lymphohistiocytosis. Haematologica. 92(7):978-981. PMID: 17606450. doi: 10.3324/ haematol.11233.

Pey, A.L., Stricher, F., Serrano, L., and Martinez, A. 2007. Predicted effects of missense mutations on native-state stability account for phenotypic outcome in phenylketonuria, a paradigm of misfolding diseases. Am. J. Hum. Genet. 81(5):1006-1024. PMID: 17924342. doi: $10.1086 / 521879$.

Ramachandran, R., Tweten, R.K., and Johnson, A.E. 2004. Membrane-dependent conformational changes initiate cholesterol-dependent cytolysin oligomerization and intersubunit beta-strand alignment. Nat. Struct. Mol. Biol. 11(8):697-705. PMID: 15235590. doi: $10.1038 /$ nsmb793.

Rameix-Welti, M.A., Regnier, C.H., Bienaime, F., Blouin, J., Schifferli, J., Fridman, W.H., SautesFridman, C., and Fremeaux-Bacchi, V. 2007. Hereditary complement C7 deficiency in nine families: Subtotal C7 deficiency revisited. Eur. J. Immunol. 37(5):1377-1385. PMID: 17407100. doi: 10.1002/eji.200636812.

Risma, K.A., Frayer, R.W., Filipovich, A.H., and Sumegi, J. 2006. Aberrant maturation of mutant perforin underlies the clinical diversity of hemophagocytic lymphohistiocytosis. J. Clin. Invest. 116(1):182-192. PMID: 16374518. doi: 10.1172/ JCI26217.

Rosado, C.J., Buckle, A.M., Law, R.H., Butcher, R.E., Kan, W.T., Bird, C.H., Ung, K., Browne, K.A., Baran, K., Bashtannyk-Puhalovich, T.A., Faux, N.G., Wong, W., Porter, C.J., Pike, R.N., Ellisdon, A.M., Pearce, M.C., Bottomley, S.P., Emsley, J., Smith, A.I., Rossjohn, J., Hartland, E.L., Voskoboinik, I., Trapani, J.A., Bird, P.I., Dunstone, M.A., and Whisstock, J.C. 2007. A common fold mediates vertebrate defense and bacterial attack. Science. 317(5844):1548-1551. PMID: 17717151. doi: 10.1126/science.1144706.

Sanchez, I.P., Leal-Esteban, L.C., Alvarez-Alvarez, J.A., Perez-Romero, C.A., Orrego, J.C., Serna, M.L., Coll, Y., Caicedo, Y., Pardo-Diaz, E., Zimmer, J., Bleesing, J.J., Franco, J.L., and Trujillo-Vargas, C.M. 2012. Analyses of the PRF1 gene in individuals with hemophagocytic lymphohystiocytosis reveal the common haplotype R54C/A91V in Colombian 
unrelated families associated with late onset disease. J. Clin. Immunol. 32(4):670-680. PMID: 22437823. doi: $10.1007 /$ s10875-012-9680-5.

Sieni, E., Cetica, V., Hackmann, Y., Coniglio, M.L., Da Ros, M., Ciambotti, B., Pende, D., Griffiths, G., and Arico, M. 2014. Familial hemophagocytic lymphohistiocytosis: When rare diseases shed light on immune system functioning. Front. Immunol. 5:167. doi: 10.3389/fimmu.2014.00167.

Sieni, E., Cetica, V., Piccin, A., Gherlinzoni, F., Sasso, F.C., Rabusin, M., Attard, L., Bosi, A., Pende, D., Moretta, L., and Arico, M. 2012. Familial hemophagocytic lymphohistiocytosis may present during adulthood: Clinical and genetic features of a small series. PLoS ONE. 7(9): e44649. PMID: 22970278. doi: 10.1371/journal.pone. 0044649.

Stefl, S., Nishi, H., Petukh, M., Panchenko, A.R., and Alexov, E. 2013. Molecular mechanisms of disease-causing missense mutations. J. Mol. Biol. 425(21):3919-3936. PMID: 23871686. doi: 10.1016/j. jmb.2013.07.014.

Stepp, S.E., Dufourcq-Lagelouse, R., Le Deist, F., Bhawan, S., Certain, S., Mathew, P.A., Henter, J.I., Bennett, M., Fischer, A., de Saint Basile, G., and Kumar, V. 1999. Perforin gene defects in familial hemophagocytic lymphohistiocytosis. Science. 286(5446):1957-1959. PMID: 10583959. doi: 10. 1126/science.286.5446.1957.

Stewart, S.E., Bird, C.H., Tabor, R.F., D’Angelo, M.E., Piantavigna, S., Whisstock, J.C., Trapani, J.A., Martin, L.L., and Bird, P.I. 2015. Analysis of perforin assembly by quartz crystal microbalance reveals a role for cholesterol and calcium-independent membrane binding. J. Biol. Chem. 290(52):31101-31112. PMID: 26542805. doi: 10.1074/jbc.M115.683078.

Tesi, B., Chiang, S.C., El-Ghoneimy, D., Hussein, A.A., Langenskiold, C., Wali, R., Fadoo, Z., Silva, J.P., Lecumberri, R., Unal, S., Nordenskjold, M., Bryceson, Y.T., Henter, J.I., and Meeths, M. 2015. Spectrum of atypical clinical presentations in patients with biallelic PRF1 missense mutations. Pediatr. Blood Cancer. 62(12):2094-2100. PMID: 26184781. doi: $10.1002 / p b c .25646$.

Thiery, J., and Lieberman, J. 2014. Perforin: A key poreforming protein for immune control of viruses and cancer. Subcell Biochem. 80197-80220. doi: 10.1007/ 978-94-017-8881-6_10.

Tokuriki, N., and Tawfik, D.S. 2009. Stability effects of mutations and protein evolvability. Curr. Opin. Struct. Biol. 19(5):596-604. PMID: 19765975. doi: 10. 1016/j.sbi.2009.08.003.
Tokuriki, N., Stricher, F., Serrano, L., and Tawfik, D.S. 2008. How protein stability and new functions trade off. PLoS Comput. Biol. 4(2):e1000002. PMID: 18463696. doi: 10.1371/journal.pcbi.1000002.

Ueda, I., Kurokawa, Y., Koike, K., Ito, S., Sakata, A., Matsumora, T., Fukushima, T., Morimoto, A., Ishii, E., and Imashuku, S. 2007. Late-onset cases of familial hemophagocytic lymphohistiocytosis with missense perforin gene mutations. Am. J. Hematol. 82(6):427-432. PMID: 17266056. doi: 10.1002/ajh.20878.

Urrea Moreno, R., Gil, J., Rodriguez-Sainz, C., Cela, E., LaFay, V., Oloizia, B., Herr, A.B., Sumegi, J., Jordan, M.B., and Risma, K.A. 2009. Functional assessment of perforin $\mathrm{C} 2$ domain mutations illustrates the critical role for calcium-dependent lipid binding in perforin cytotoxic function. Blood. 113(2):338-346. PMID: 18927437. doi: 10.1182/ blood-2008-08-172924.

Voskoboinik, I., Dunstone, M.A., Baran, K., Whisstock, J.C., and Trapani, J.A. 2010. Perforin: Structure, function, and role in human immunopathology. Immunol. Rev. 235(1):35-54. PMID: 20536554. doi: 10.1111/j.0105-2896.2010.00896.x.

Voskoboinik, I., Whisstock, J.C., and Trapani, J.A. 2015. Perforin and granzymes: Function, dysfunction and human pathology. Nat. Rev. Immunol. 15(6):388400. PMID: 25998963. doi: 10.1038/nri3839.

Wang, Y., Wang, Z., Chen, H., and Wang, X. 2012. Adult onset of primary hemophagocytic syndrome in subjects carrying PRF1 mutations. Ann. Hematol. 91:1489-1490. doi: 10.1007/s00277-012-1409-2.

Wang, Z., and Moult, J. 2001. SNPs, protein structure, and disease. Hum. Mutat. 17(4):263-270. PMID: 11295823. doi: 10.1002/humu.22.

Worth, C.L., Preissner, R., and Blundell, T.L. 2011. SDM-A server for predicting effects of mutations on protein stability and malfunction. Nucleic Acids Res. 39(Web Server issue):W215-W222. PMID: 21593128. doi: 10.1093/nar/gkr363.

Yagi, H., Conroy, P.J., Leung, E.W., Law, R.H., Trapani, J.A., Voskoboinik, I., Whisstock, J.C., and Norton, R.S. 2015. Structural basis for $\mathrm{Ca}^{2+}$-mediated interaction of the Perforin C2 Domain with Lipid membranes. J. Biol. Chem. 290(42):25213-25226. PMID: 26306037. doi: 10.1074/jbc.M115.668384.

Yin, S., Ding, F., and Dokholyan, N.V. 2007. Eris: An automated estimator of protein stability. Nat. Methods. 4(6):466-467. PMID: 17538626. doi: 10. 1038/nmeth0607-466.

Yue, P., Li, Z., and Moult, J. 2005. Loss of protein structure stability as a major causative factor in monogenic 
disease. J. Mol. Biol. 353(2):459-473. PMID: 16169011. doi: 10.1016/j.jmb.2005.08.020.

Zhang, K., Jordan, M.B., Marsh, R.A., Johnson, J.A., Kissell, D., Meller, J., Villanueva, J., Risma, K.A., Wei, Q., Klein, P.S., and Filipovich, A.H. 2011. Hypomorphic mutations in PRF1, MUNC13-4, and STXBP2 are associated with adult-onset familial HLH. Blood. 118(22):5794-5798. doi: 10.1182/ blood-2011-07-370148.
Zur Stadt, U., Beutel, K., Kolberg, S., Schneppenheim, R., Kabisch, H., Janka, G., and Hennies, H.C. 2006. Mutation spectrum in children with primary hemophagocytic lymphohistiocytosis: Molecular and functional analyses of PRF1, UNC13D, STX11, and RAB27A. Hum. Mutat. 27(1):62-68. PMID: 162 78825. doi: 10.1002/humu.20274. 